\title{
Animação e Rock'n'Roll Usados para Facilitar o Ensino de Astronomia e Química
}

\author{
Jane Gregorio-Hetem* \\ Departamento de Astronomia do Instituto de Astronomia, Geofísica e Ciências Atmosféricas \\ da Universidade de São Paulo \\ * Autor para correspondência: jane.gregorio.hetem@iag.usp.br
}

\section{RESUMO}

Por sua característica multidisciplinar, a Astronomia tem sido amplamente utilizada na aplicação da pesquisa científica no ensino de ciências naturais. As chamadas por projetos de ensino e divulgação científica, visando à produção de materiais didáticos, configuram-se como excelentes oportunidades de aliar o fascínio da população pelos astros e a informação que estes podem trazer sobre conceitos básicos das ciências fundamentais. No presente trabalho, descrevemos a experiência de criar materiais didáticos em linguagem atrativa para os jovens (livros de história em quadrinhos, filmes de animação), com o objetivo de ampliar a cultura científica entre o público em geral. Para os estudantes da graduação, nossos projetos fazem parte das ações afirmativas que buscam diminuir a evasão e estimular a conclusão do curso no tempo ideal. Este artigo é dedicado à descrição da metodologia adotada e à apresentação de alguns exemplos dos resultados alcançados nesse tipo de atuação, "antes" e "depois" do ingresso do estudante na graduação.

Palavras-chave: Difusão Científica; Materiais Didáticos: Animação; Ensino de Astronomia: Estrutura e Evolução Estelar; Ensino de Química: Átomos e Moléculas.

\begin{abstract}
Due to its multidisciplinary character, astronomy has been subject of educational projects that use scientific research to improve the learning of natural sciences. The financial support in the form of Announcements of Opportunities, devoted to the production of teaching and outreach material, are the best way to combine the fascination that people have about the stars, with the information that these objects can bring concerning the basic concepts related to fundamental sciences. In the present work, we describe the experience in developing educational resources by adopting modern communication language (comics, animations) that is attractive among young people. Our goal is to enlarge the scientific culture of the citizens. For undergraduate students, our projects aim to diminish the evasion, as well as to promote the course conclusion in the ideal period. The paper is dedicated to describe the adopted methodology and to report the partial results of acting "before "and "after" the student entrance in the undergraduate course.
\end{abstract}

Keywords: Outreach; Educational Resources: Animation; Astronomy Teaching: Stellar Structure and Evolution; Chemical Teaching: Atoms and Molecules.

\section{Introdução}

Motivados pelo crescente interesse em estreitar os laços entre a comunidade científica e o público em geral, um grande número de universidades e de centros de pesquisa tem se dedicado ao aperfeiçoamento de métodos educacionais. Nos cursos relacionados às ciências fundamentais, dentre os maiores desafios enfrentados pelas comissões de graduação e/ou coordenação de curso está a procura por soluções para evitar a evasão ou o tempo prolongado de conclusão. Isso ocorre tanto nas universidades brasileiras como no cenário internacional.

Nos últimos oito anos, desde a criação do Bacharelado em Astronomia no IAG, temos procurado aproveitar as diversas oportunidades de fomento que nossa Universidade vem oferecendo, em editais como: Ensinar com Pesquisa, Aprender com Cultura e Extensão, PEEG e, em particular, o Programa Pró-Ensino, ao qual se refere o presente 
trabalho, para implementar ações voltadas à melhoria do ensino e aprendizado em nossa área.

Com o principal objetivo de combater a evasão e promover a conclusão do curso no tempo ideal, temos aplicado a multidisciplinaridade intrínseca à Astronomia, incluindo seus aspectos científicos, tecnológicos e culturais, em projetos que visam a: (i) divulgar a ciência através de uma conscientização do atual estágio de conhecimento e das ferramentas de que se dispõe para a compreensão do Universo; e (ii) elaborar novos materiais com a proposta de divulgar a Astronomia e ao mesmo tempo ensinar conceitos básicos de Ciências Exatas. Tais propostas fazem parte das ações afirmativas do Departamento de Astronomia, coordenadas pela Comissão Coordenadora do Curso de Graduação e Comissão de Cultura e Extensão, que intentam garantir as melhores condições de aprendizado, atuando "antes, durante e depois" do ingresso do aluno em nosso bacharelado. Dessa forma, ao mesmo tempo que oferecemos ao público geral um maior conhecimento da atuação profissional relacionada com nossa área, visamos também aos professores e aos estudantes pré-vestibulandos, buscando despertar o interesse do jovem por ciência e pesquisa científica. Trata-se de ações baseadas no fato de que os alunos devem estar bem preparados para a aquisição dos conteúdos propostos no Projeto Pedagógico, que envolvem uma forte base em Astronomia, Física, Matemática, e Computação. Nesse sentido, torna-se necessária a correta divulgação do curso e da profissão do astrônomo, para atrair estudantes com as habilidades requeridas. Após o ingresso, as ações voltadas aos graduandos do nosso bacharelado procuram estimular o aprendizado por meio de atividades práticas e projetos que aproximem o estudante da realidade profissional do astrônomo.

No presente trabalho, descrevo nossa experiência na realização de projetos voltados ao ensino e à divulgação científica, em particular na produção de material didático, por meio de projetos anteriores, em editais do GNPq (Seções 1.1 e 1.2), e mais recentemente pelo Programa Pró-Ensino da Pró-Reitoria de Graduação da USP. A seguir, apresentam-se o desenvolvimento e os resultados do projeto, que se encontra em andamento. Ao final, ganham espaço as conclusões parciais.

\section{Ombros de Gigantes: 2009 - Ano Internacional da Astronomia}

Na ocasião do Ano Internacional da Astronomia, o GNPq lançou um edital visando à popularização da Astronomia, por meio da produção de materiais didáticos e de difusão científica, entre outros.

No âmbito desse edital do CNPq, elaboramos o livro Ombros de Gigantes: a História da Astronomia em Quadrinhos (GREGORIO-HETEM, HETEM JUNIOR \& TENÓRIO, 2009), com o principal objetivo de ensinar conceitos básicos de Física, usando a Astronomia como o ponto de partida para estimular nos jovens o interesse pela ciência. Nesse livro, o processo de aprendizagem se dá pela forma lúdica (história em quadrinhos), em nível de excelência (textos complementares formais) e prática (sugestão de experimentos relacionados, com materiais de baixo custo).

Contamos com o apoio da Universidade Estadual do Rio de Janeiro (UERJ), para a distribuição gratuita dos livros nas escolas participantes da Olimpíada Brasileira de Astronomia (OBA). Graças a esse projeto do CNPq, e à colaboração de colegas da UERJ, foram produzidos 16500 exemplares do livro para distribuição gratuita a professores e ao público em geral, participantes de eventos de divulgação científica, além dos participantes da OBA. Parte desses exemplares foi destinada para distribuição nos Cursos de Extensão Universitária do IAG/USP, bem como para estudantes de graduação.

Um interessante depoimento do jovem Allan Costa, medalhista da $8^{\text {a }}$ Olimpíada Internacional de Astronomia e Astrofísica, realizada em Suceava, cidade da Romênia, aparece no vídeo que ele produziu visando à participação num evento promovido pelo European Southern Observatory (COSTA, 2014). Nesse vídeo, que o jovem descreve como "[...] is the application for the ESO Camp. It is also a tribute to the many great adventures that Astronomy has provided me those last years", nosso livro é citado entre os diversos fatores que estimularam seu interesse por 
Astronomia. Suas experiências o levaram a atuar no projeto educacional Além da Lousa, buscando levar aos estudantes do Ensino Médio o interesse pelo aprendizado científico, que pode ser aplicado em situações cotidianas (PROJETO, 2014). Consideramos esse um belo exemplo de contribuição da divulgação científica para promover melhorias de ensino em nosso país.

\section{A Química e os Astros:2011 -Ano Internacional da Astronomia}

A partir da experiência de nossa equipe em produzir o livro Ombros de Gigantes, e inspirados pelo sucesso de sua distribuição, surgiu a motivação de inscrever um projeto para o edital CNPq lançado para o Ano Internacional da Química. Nesse caso, em vez de história em quadrinhos, a parte lúdica foi apresentada na forma de animações, e a temática foi voltada aos conceitos relacionados com as espécies atômicas e moleculares, buscando estimular o interesse dos estudantes e do público em geral pela ciência química.

Em um programa da série Educação Brasileira, veiculado pela Univesp TV, nosso colega Guilherme Marson, do IQ/USP, descreve o impacto esperado no Ano Internacional da Química. Além de destacar a importância da popularização da Química, nessa entrevista o professor Marson ressalta o papel do ensino de Química durante a educação básica para a formação do cidadão (MARSON \& GRANETTO, 2011).

A interdisciplinaridade envolvendo conceitos de Química apresentados do ponto de vista astronômico é uma excelente oportunidade para explorar questões fundamentais da humanidade. A população em geral precisa e quer saber mais sobre nossas origens e nosso destino, e também sobre as presentes condições que garantem a existência da vida como a conhecemos. Dessa forma, adotamos o contexto do ciclo de vida das estrelas: nascimento, maturidade e morte, para descrever: (i) a origem dos elementos químicos, que também se relaciona com a produção de energia por processos termonucleares que ocorrem no interior de estrelas como nosso Sol; e (ii) a presença de moléculas no meio interestelar, em particular nas regiões de formação estelar, que podem ser quimicamente enriquecidas pela ocorrência de uma explosão de supernova (o fim catastrófico de uma estrela de alta massa).

Parte desse projeto foi executada com apoio do $\mathrm{CNPq}$, que levou à produção de dois filmes de animação, e a parte complementar foi contemplada pelo Programa Pró-Ensino 2013, no projeto intitulado "A Química e as estrelas: a Origem e a Presença das Espécies Químicas no Universo", para o desenvolvimento do terceiro filme da série, descrito a seguir.

\section{Desenvolvimento: uma História sobre a Origem e a Presença das Espécies Químicas no Universo}

Para despertar entre os estudantes o interesse pelas Ciências Moleculares, a partir de nossa experiência em pesquisa, ensino e divulgação, realizamos um trabalho baseado na interdisciplinaridade que associa a Astronomia com outras áreas, em particular a Química. Visando a um produto a ser difundido por um moderno meio de comunicação, que é a linguagem da animação, o projeto foi elaborado para atender pelo menos quatro das linhas de ação consideradas no Edital Pró-Ensino: (i) aproximação ainda maior de pesquisa e ensino; (ii) produção de materiais didáticos com linguagens diversas; (iii) elaboração de materiais didáticos multidisciplinares; e (iv) divulgação em meios eletrônicos contribuindo para a divulgação do conhecimento gerado na nossa universidade.

Além de contribuir para o preenchimento de uma lacuna na difusão do conhecimento, nossa proposta buscou abrir caminhos para a interdisciplinaridade, que permite ampliar a cultura científica, tão necessária para a cidadania.

A moderna linguagem das animações, que se configura em um veículo de importante inserção entre os jovens, foi adotada como meio de assimilação e difusão de informação, para apresentar conceitos básicos de Química relacionados com o ciclo de vida das estrelas e a evolução química da Galáxia.

Os temas abordados foram distribuídos de forma crescente em termos de complexidade das espé- 
cies químicas. Assim, o primeiro filme de animação versou sobre os elementos químicos, o segundo sobre moléculas simples, e o terceiro sobre moléculas complexas e compostos pré-bióticos.

\section{Metodologia}

Os métodos modernos aplicados no processo de aprendizagem estão diretamente vinculados à pesquisa científica. As novas técnicas e metodologias de ensino implicam desafios tanto para educadores como para pesquisadores, na busca por uma maior integração das descobertas científicas no cotidiano dos jovens e do público em geral. No Brasil, em particular, tais desafios são amplificados pelas dificuldades inerentes à falta de recursos para a educação e aos problemas sociais que o país enfrenta, principalmente nas regiões menos favorecidas.

Por outro lado, as facilidades de acesso aos diversos meios de comunicação e diversão, como televisão, internet, jogos eletrônicos etc., induzem nos jovens o desinteresse por valores importantes no processo de aprendizado, como a leitura e a reflexão. Assim, o educador enfrenta dificuldades adicionais relacionadas com a necessidade de captar a atenção de seus estudantes e despertar seu interesse pelos conteúdos programáticos.

Nesse sentido, para promover o aprendizado de conceitos básicos de Química de forma atrativa e estimulante, propomos a apresentação do material didático por meio de animações voltadas ao público jovem, cujo personagem principal é um adolescente apaixonado por música (Rock'n'Roll) e descobertas científicas.

O recurso pedagógico adotado visa a utilizar a linguagem da ficção, que tem forte apelo entre os jovens, para facilitar o aprendizado de conceitos relacionados à interpretação da Tabela Periódica, que são conteúdos programáticos geralmente considerados pelos estudantes como pouco atrativos ou de difícil assimilação. A Figura 1 ilustra o personagem principal e o cenário adotado, mostrando as características jovial e lúdica das animações.

Os temas abordados no filme 1, Rockstar e a Origem do Metal, envolvem: estrutura atômica; fusão nuclear e nucleossíntese; estrutura estelar: o interior do Sol; composição química do Sol; a produção de energia solar; estágios finais da evolução do Sol; a origem dos metais pesados; formação do Sistema Solar; e o ciclo de vida das estrelas. Dado o grande volume de informações apresentadas de forma condensada, foi disponibilizado na internet um livreto explicativo (GREGORIO-HETEM \& HETEM JUNIOR, 2012) com informações detalhadas sobre os conceitos que se pretende ensinar, conforme ilustrado na Figura 2.

Essa é uma importante característica do projeto, cuja prática ensino/aprendizado segue três etapas básicas: a animação desperta o interesse e a atenção do jovem, através da abordagem cotidiana; o texto complementar apresenta a descrição teórica e mais aprofundada dos temas, estimulando assim um aprendizado pela "apreensão" em vez de memorização.

Os conteúdos das cartilhas associadas aos filmes 2 e 3 já foram elaborados e encontram-se em fase de diagramação e ilustração. Uma parte desses textos foi produzida com a participação de estudantes de graduação, sendo uma delas bolsista do programa Pró-Ensino, da Universidade de São Paulo.

\section{Resultados}

O projeto aprovado pelo referido Programa Pró-Ensino da USP foi elaborado para realizar o último filme de uma série de três animações, denominado Rockstar e as Moléculas no Espaço. Trata-se da continuação do projeto anterior, financiado pelo CNPq, no âmbito do Ano Internacional da Química, como parte de um programa de elaboração de materiais educacionais alternativos. A verba do CNPq permitiu a produção apenas dos dois primeiros filmes, os quais já foram lançados e disponibilizados na internet: Rockstar e a Origem do Metal e Rockstar e o Mistério da Água. Até o momento, a contagem de acessos ao filme 1 é de cerca de 35600 e do filme 2 é aproximadamente 5800. Os comentários apresentados pelo público são muito positivos, confirmando o sucesso do projeto. Tais filmes também fazem parte da programação de apresentação contínua no museu de ciências 
Catavento Cultural Educacional, com sede no Palácio das Indústrias, localizado no centro da cidade de São Paulo.

Para concentrar as informações e o material produzido, preparamos uma página $w e b^{1}$, onde mais informações podem ser obtidas, assim como
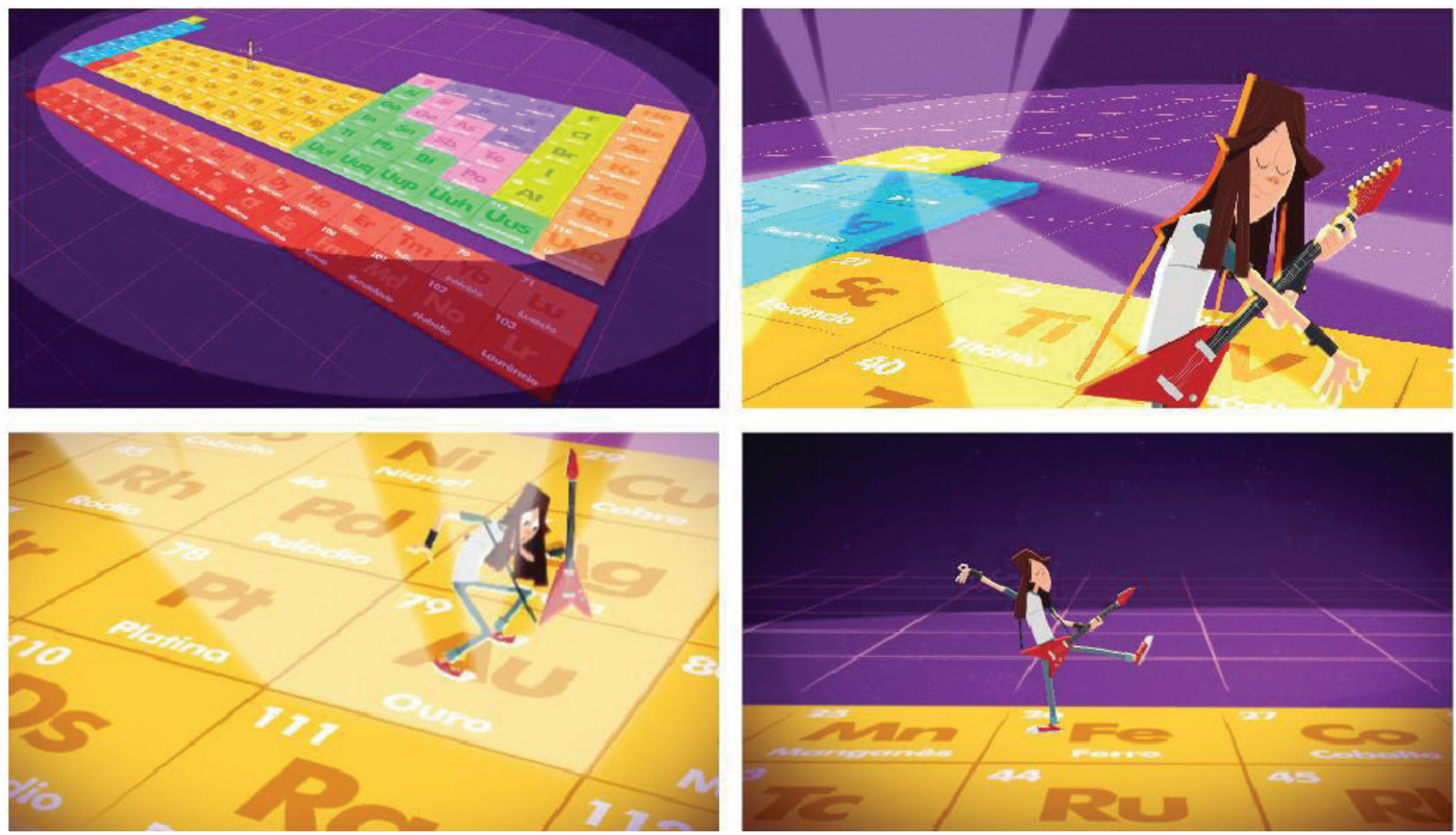

Figura 1 - Exemplos de cenários compostos para o filme Rockstar e a Origem do Metal, tendo como base a Tabela Periódica.

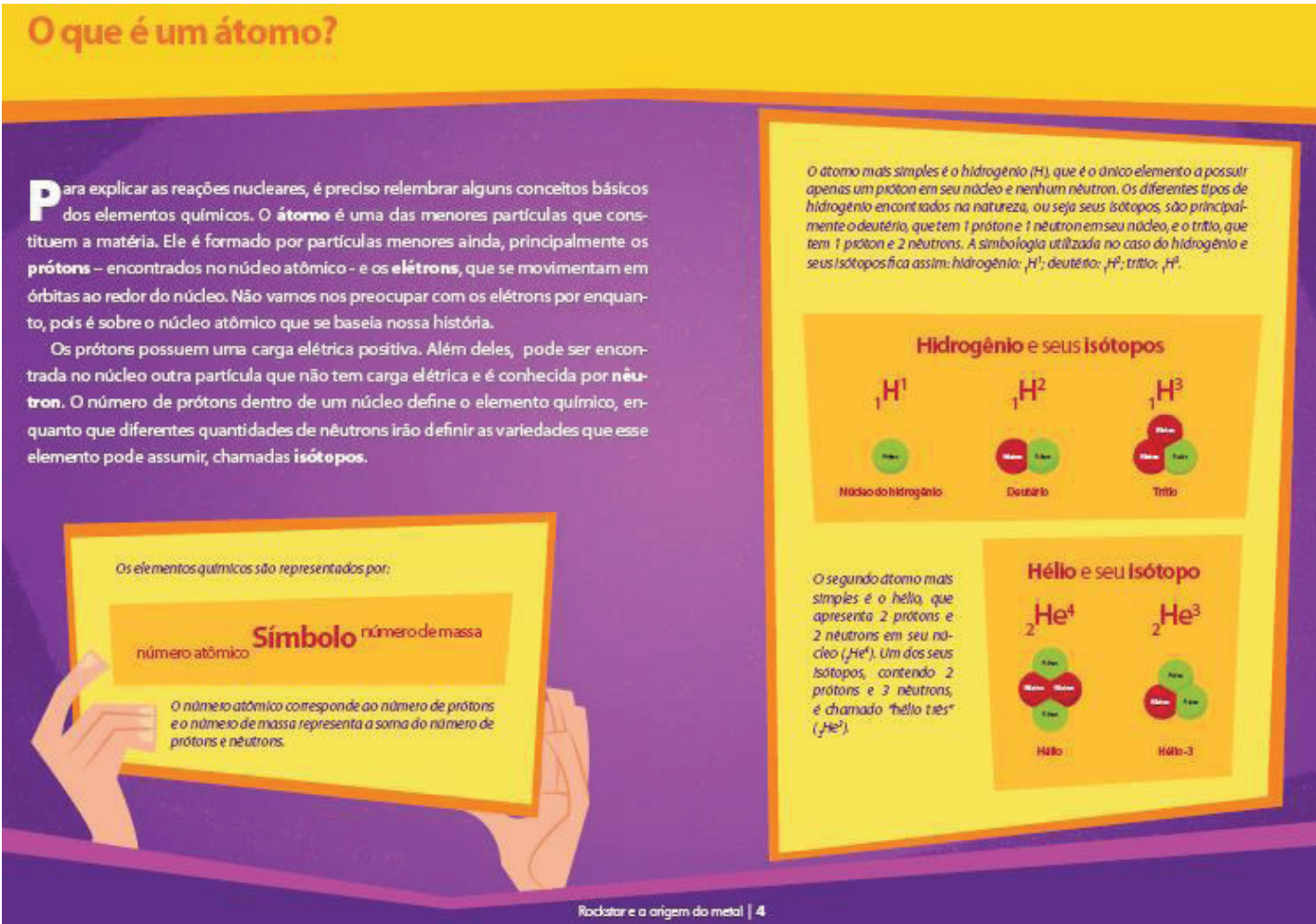

Figura 2 - Extrato do livreto explicativo (cartilha em pdf disponibilizada na internet) com texto mais detalhado a respeito de um dos temas do filme Rockstar e a Origem do Metal. 


\section{Conclusões Parciais}

O desenvolvimento do filme 3 ainda está em andamento, sendo que apenas a etapa de pré-produção (roteiros, dublagem e storyboard) foi realizada, e ainda aguardamos outras fontes de financiamento necessárias para sua finalização. Mesmo assim, os resultados anteriores, bem como aqueles descritos no presente trabalho, demonstram o alcance dos objetivos propostos.

Nosso principal interesse foi despertar o gosto pela Química por meio de uma apresentação lúdica, permitindo aos jovens que se sintam estimulados a aprofundarem seus conhecimentos nessa área. Este material pode contribuir para o ensino de alguns tópicos de disciplinas de graduação oferecidas pelo Departamento de Astronomia, tais como AGA0215 - Fundamentos de Astronomia, entre outras que tenham como parte dos conteúdos a composição química do Sistema Solar e do Meio Interestelar. Os conteúdos podem também ser abordados em AGA0210 - Introdução à Astronomia (para alunos de outros cursos, das áreas biológicas e de humanidades); AGA0421 - Divulgação em Astronomia, e mesmo em disciplinas da pós-graduação, como MPA5026 - Tópicos Avançados em Astronomia e Divulgação Científica, do Mestrado Profissional em Ensino de Astronomia.

Para a estudante de graduação que foi bolsista do projeto, a participação na elaboração de textos, para detalhamento dos conteúdos que serão incluídos no folheto explicativo do filme 3 , foi uma excelente oportunidade para também se aprofundar no tema, a partir de pesquisa bibliográfica e redação de textos, bem como desenvolver habilidades de divulgação de materiais didáticos na internet.

É interessante notar que, antes de participar do projeto, a estudante cogitou a possibilidade de mudar de curso, pois, apesar de seu bom desempenho nas disciplinas da área de exatas, ela notou que sua vocação estava mais voltada para a área de humanidades, com particular interesse em Letras. A participação no projeto permitiu a aplicação de suas habilidades em redação de textos de divulgação. Além de estimular sua permanência no curso, sua dedicação ao projeto levou a estudante à escolha do tema "Instrumentação Astronômica Antiga" para desenvolvimento de seu Trabalho de Graduação. O sucesso de sua monografia de conclusão de curso, por sua vez, inspirou a aluna a se inscrever em duas universidades na Inglaterra, para realizar o mestrado em História da Astronomia, tendo sido aceita em ambas. Esse é mais um exemplo de como projetos interdisciplinares podem em muito contribuir para a diminuição da evasão, nos casos de alunos que eventualmente não se interessem em prosseguir na mesma área da carreira escolhida na graduação, sem necessariamente terem que desistir do curso.

O outro exemplo citado, depoimento do medalhista da OBA, mostra a importância da popularização científica, visando ao engajamento do jovem na ciência, para despertar vocações para uma carreira na área das ciências fundamentais ou para estimular o espírito crítico, que advém de uma cultura científica aprofundada a ser promovida entre o público em geral.

A fim de realizar uma avaliação estatística do impacto do nosso material didático, pretendemos anexar aos livretos um questionário com perguntas gerais e específicas. O questionário, com base em algumas poucas questões, será elaborado de forma a evidenciar os aspectos positivos e negativos do material, e a sua contribuição para a melhoria da educação científica. Solicitaremos aos leitores que encaminhem suas respostas por e-mail, para que possamos, em uma eventual oportunidade, produzir uma segunda versão melhorada do material e/ou produzir outras séries de conteúdos, baseados na experiência adquirida.

\section{Nota}

1 A página web do projeto é: www.HQastroRock.iag.usp.br.

\section{Bibliografia}

"ANIMAÇÃO e Rock'n'Roll São Usados para Ensinar Astrofísica a Jovens". Estadão.com.br/Educação, 3 set. 2012. Disponível em: http://www.estadao.com. $\mathrm{br} /$ noticias/vidae, animacao-e-rocknroll-sao-usado s-para-ensinar-astrofisica-a-jovens, 925394,0.htm.

GREGORIO-HETEM, Jane \& HETEM JUNIOR, Annibal. Rockstar e a Origem do Metal: uma História 
sobre os Átomos e as Estrelas. Projeto gráfico e ilustrações de Marlon Tenório. São Paulo: Instituto de Astronomia, Geofísica e Ciências Atmosféricas, 2012. Disponível em: http://www.iag.usp.br/astronomia/sites/default/files/ cartilha_diagramada09_web.pdf.

GREGORIO-HETEM, Jane; HETEM JUNIOR, Annibal \& TENÓRIO, M. Ombros de Gigantes: a História da Astronomia em Quadrinhos. São Paulo: Instituto de Astronomia, Geofísica e Ciências Atmosféricas da Universidade de São Paulo, 2009.

PROJETO Além da Lousa Desperta Interesse Científico e Leva Estudantes para Fora da Sala de Aula. Cotidiano.UFSC, 25 nov. 2014. Disponível em: http:// cotidiano.sites.ufsc.br/projeto-alem-da-lousa-despertainteresse-cientifico-e-leva-estudantes-para-fora-da-salade-aula/.

VÍDEO Procura Facilitar Ensino de Astronomia e Química. Agência USP de Notícias, 3 set. 2012. Disponível em: http://www.usp.br/agen/?p=110539.

\section{Filmes}

Filme 1: Rockstar e a origem do Metal. Disponível em: https://www.youtube.com/watch?v=wIEhSIt1oEI.

Filme 2: Rockstar e o Mistério da Água. Disponível em: https://www.youtube.com/watch?v=f4WR73u0Dyc.

\section{Vídeos}

COSTA, A. Vídeo produzido para o ESO Camp, promovido pelo European Southern Observatory, 2014. Disponível em: https://www.youtube.com/ watch?v $=9$ WeuvcAayYg.

GRANETTO, E. "A Origem do Metal (Ederson Granetto entrevista Jane e Annibal Hetem)". Notícias Univesp, Univesp TV, 13 set. 2012. Disponível em: http://www.youtube.com/watch? $\mathrm{v}=1 \mathrm{iRCuB} 5 \mathrm{~s}_{-}$ $\mathrm{xU} \&$ feature $=$ plcp.

MARSON, G. A. \& GRANETTO, E. "Entrevista sobre o Significado do Ano Internacional da Química". Educaşão Brasileira 32, Univesp TV, 13 jun. 2011. Disponível em: https://www.youtube.com/ watch? $=\mathrm{G} 5 \mathrm{O} \_\mathrm{XgSVx} 0$. 